\title{
Prevalence of surreptitious laxative abuse in patients with diarrhoea of uncertain origin: a cost benefit analysis of a screening procedure
}

\author{
P BYTZER, M STOKHOLM, I ANDERSEN, N A KLITGAARD, \\ AND O B SCHAFFALITZKY DE MUCKADELL
}

From the Department of Medical Gastroenterology and the Department of Clinical Chemistry, Odense University Hospital, Odense, Denmark

SUMmARY The costs and medical benefits of an early, routine laxative screening test in patients with diarrhoea of uncertain origin was evaluated. During a two year period 200 consecutive, unselected patients complaining of diarrhoea were considered for the study in whom a three day faecal collection was undertaken. Fifty four patients denying laxative consumption had diarrhoea (mean daily stool weight $>200 \mathrm{~g}$ ) of uncertain origin at their initial visit of whom 47 were screened to detect ingestion of anthraquinones, bisacodyl, phenolphthalein, and magnesium salts. Seven patients had positive tests. No single clinical feature could have predicted the outcome of the test. The possible cost savings of the programme were estimated by not releasing the results of the test to the clinicians until the patient's investigations were complete. The seven patients with laxative abuse spent a total of 35 days in hospital and were seen on 29 occasions in the outpatient clinic after the laxative screening test was positive. The cost of the screening programme was cheaper than the costs of the diagnostic procedures in patients with laxative abuse. We recommend the use of a comprehensive, early laxative screening programme in all patients with diarrhoea of uncertain origin as a cost effective procedure.

Patients who surreptitiously ingest laxatives in order to cause diarrhoea represent a special diagnostic challenge to the clinical gastroenterologist. Many patients undergo expensive, unnecessary, and even risky investigations before the diagnosis is considered. ${ }^{1-3}$

The diagnosis can be confirmed either by a screening for laxatives in the patients faeces ${ }^{45}$ or by a search of the patient's room and belongings. ${ }^{\circ}$ Both methods, however, imply a suspicion of drug abuse, and unfortunately this suspicion often arises very late in the diagnostic process, and is usually provoked by the negative outcome of an extensive workup. ${ }^{7-9}$

Address for correspondence: Dr P Bytzer. Department of Medical Gastroenterology S, Odense University Hospital, DK-50(0) Odense C. Denmark.

Accepted for publication 9 February 1989
A toxicological screening for laxatives in all patients with diarrhoea of uncertain origin, at an early stage, might be cost effective provided that the condition is not extremely uncommon. Unfortunately, valid data on the prevalence rate of laxative abuse among unselected patients are lacking, but the syndrome has been considered the leading cause of chronic diarrhoea of unknown origin in patients studied prospectively."

In order to determine the prevalence rate of concealed laxative drug consumption, we decided to construct a comprehensive screening programme, taking into account the diversity of available laxative preparations.

In this paper, we present the results of a prospective two year laxative screening test on consecutive patients with diarrhoea of uncertain origin and a costbenefit analysis of the screening programme. 


\section{Methods}

PATIENTS

Over a two year period (1986-88) all patients referred to the Department of Medical Gastroenterology S, Odense University Hospital, because of diarrhoea were candidates for the study. The department serves a population of 500000 in the county of Funen, and receives approximately 2000 new patients a year.

Before entry each patient was interviewed and symptoms related to the bowel disorder were recorded. A three day quantitative stool collection without marker correction and without dietary advice or control was completed and patients with stool weights $>200 \mathrm{~g}$ per day were questioned about laxative consumption. Patients denying ingestion of laxatives were asked to participate in the study, and informed that the diagnostic programme would include tests of urine and faeces for substances that might influence stool weight. The patients were informed that they had the right to refuse. Patients with normal stool weight or with diarrhoea of obvious - for example, profuse bloody diarrhoea - or well known origin, and patients with steatorrhoea more than $10 \mathrm{~g}$ of fat per day were not included in the study. The study was conducted in accordance with the Helsinki II declaration and was approved by the regional ethics committee for Funen and Vejle counties.

\section{SCREENING PROGRAMME}

In the study period 41 different laxatives were registered for over the counter sale in Denmark. Twenty seven drugs contained stimulant laxatives (anthraquinones or bisacodyl and combinations of these with phenolphthalein). The main active ingredient was mineral oils with trace amounts of phenolphthalein in three drugs. Two preparations contained lactulose, and two contained magnesium salts. One contained dioctylsodium sulphosuccinate and the remaining were dominated by bulk producing ingredients. A preparation containing potassium and sodium sulphates as the only laxative ingredients was registered in the first part of the study period only. During this period approximately 500 packages of the drug were sold per year. Consequently, an analysis of sulphates in faecal water was not included in the programme. Stimulant laxatives and preparations containing magnesium salts accounted for $70 \%$ of the total sale defined as equipotent doses. Based on these facts we decided to construct a screening programme to detect excretion of bisacodyl, phenolphthalein, and anthraquinones in urine and excessive magnesium in faecal water.

Screening was planned for all patients, as soon as they entered the study. Ideally three screening episodes were to be done in all patients with continuing diarrhoea, as long as the bowel disorder was unexplained. Screening was done on different days and was postponed for one week in patients who received laxatives before diagnostic procedures (barium enema, colonoscopy etc).

URINEAND FAECAL WATER ANALYSES

At each screening episode $50 \mathrm{ml}$ urine was collected and kept at $-20^{\circ} \mathrm{C}$ until analysed to detect ingestion of anthraquinones, bisacodyl, and phenolphthalein. ${ }^{+}$ "'Briefly, $20 \mathrm{ml}$ urine was adjusted to $\mathrm{pH} 5$ with $0 \cdot 1 \mathrm{M}$ hydrochloric acid, added $2 \mathrm{ml}$ acetate buffer $\mathrm{pH} 5$ and 10000 Fishmann units of mixed glucuronidase/ sulphate (from Helix pomatia, Sigma) and left overnight at $37^{\circ} \mathrm{C}$. The urine was poured on to a Tox Elut $^{\circledR}$ column (Merck) and eluted with a mixture of chloroform:isopropanol 9:1. The eluate was evaporated to dryness under a stream of nitrogen and the residual taken up in $100 \mu \mathrm{l}$ chloroform before thin layer chromatography (TLC).

The TLC was performed using silica gel coated plates (HPTLC, Merck) with fluorescent indicator and concentration zone. As mobile phases we used m-xylene: isobutyl methyl ketone: methanol 10:10:1 and hexane:toluene:acetic acid 3:1:1. The compounds were located by immersing the plates into a 6 $M$ sodium hydroxide solution. As reference compounds we used phenolphthalein, bisacodyl, danthron, and an extract of senna leaves.

The qualitative test for anthraquinone, bisacodyl and phenolphthalein ingestion has been validated previously. ${ }^{4}$ "Anthraquinones was found in all urine specimens from 16 patients known to take anthraquinone containing laxatives in doses ranging from one to six tablets a day. All urine tests made before laxative ingestion were negative. ${ }^{+}$During multiple dosing anthraquinones could be detected in urine until 96 hours afteringestion of the last dose. ${ }^{4}$ At least 32 hours after a single dose of bisacodyl, phenolphthalein, or anthraquinone the drug could be detected in the urine. None of 73 non-laxative drugsinterfered with the detection."

In the case of fluid or semifluid faeces $10-50 \mathrm{ml}$ stool water was collected and $\mathrm{pH}$ and osmolality (5100 Vapor pressure osmometer, Wescor Inc) were measured. In addition sodium, potassium, and magnesium concentrations were obtained using flame photometry and atomic absorption spectrophotometry.

COST BENEFIT ANAI.YSIS

In an attempt to evaluate the possible costs and medical benefits of a routine laxative screening programme the results of the present laxative screening were not disclosed to the participating clinicians 
until after the patient had finished the diagnostic workup, and a definitive or provisional diagnosis had been established. For all patients with a positive laxative screening test the monetary costs of diagnostic procedures aimed at the bowel disorder, and the monetary costs of days in hospital and ambulatory visits that were potentially unnecessary - that is, had been carried out after the first positive screening test, were compared with the monetary costs of screening in the entire population of patients with diarrhoea. Prices are given in Danish kroner (DKK) (£1 sterling $=13$ DKK).

In this analysis the estimated additional costs of a positive test - for example, additional screening episodes, were not included, as they were thought to vary for different 'types' of surreptitious laxative abusers.

\section{STATISTICAL ANALYSIS}

The statistical methods used were Fisher's exact test and the Mann-Whitney U test. Only two-tailed tests were used. Values of $\mathrm{p}<0.05$ were considered significant.

\section{Results}

During the study period 200 patients complaining of diarrhoea were considered for the study. Fifty four patients ( 34 women) had stool weights $>200 \mathrm{~g} /$ day without an obvious cause. Of the remaining 146 patients, 138 had normal stool weight and eight patients had significant steatorrhoea. Seven of the 54 patients with diarrhoea of uncertain origin at first visit were not screened: in two patients (one woman) the symptoms disappeared before screening could be done, four women refused to participate, and one woman was not investigated by a mistake.

Thus 47 patients ( 28 women) were screened for evidence of laxative abuse during the study period. Urine was examined in 47 patients (median two episodes, range 1-7). Stoolwater was investigated in 12 patients. In the remaining patients the test was made impossible by stool consistency.

Seven patients $(15 \%)$ had positive tests on one or more occasions. In five patients an anthraquinone excretion product was found in the urine at $1 / 2,2 / 7,3 / 3$, $1 / 1$, and $1 / 3$ screening episodes, respectively. In two patients bisacodyl was detected in the urine at $1 / 1$ and $1 / 3$ screening episodes, respectively.

No differences in faecal water composition were observed between laxative abusers and the others in the 12 patients so studied, with the single exception of a high $\mathrm{Mg}++$ output of $31 \mathrm{mmol} /$ day (normally $<20 \mathrm{mmol} / \mathrm{day}$ ). ${ }^{8}$ This suggested ingestion of a magnesium containing purgative in a female patient, who was also found to ingest bisacodyl at a different screening episode.
Table 1 Characteristics of patients screened for laxative abuse (range in parentheses)

\begin{tabular}{|c|c|c|c|}
\hline & $\begin{array}{l}\text { Positive test } \\
n=7\end{array}$ & $\begin{array}{l}\text { Negative test } \\
n=40\end{array}$ & $p$ value \\
\hline Sex ratio (M:F) & $1: 6$ & $18: 22$ & NS \\
\hline Median age $(y r)$ & $43(21-74)$ & $46(15-75)$ & NS \\
\hline $\begin{array}{l}\text { Median faecal weight, } \\
\text { g/day }\end{array}$ & $523(219-1200)$ & $297(205-2075)$ & NS \\
\hline $\begin{array}{l}\text { Median maximal bowel } \\
\text { movements, } n / \text { day }\end{array}$ & $6(2-15)$ & $6(2-40)$ & NS \\
\hline $\begin{array}{l}\text { Median length history } \\
\text { (mths) }\end{array}$ & $10(5-420)$ & $10 \cdot 5(0 \cdot 3-360)$ & NS \\
\hline Nocturnal diarrhoea (n) & $5 / 7$ & $17 / 40$ & NS \\
\hline Median stool consistency* & $4(3-4)$ & $4(1-4)$ & NS \\
\hline Psychiatric disorders (n) & $4 / 7$ & $4 / 40$ & $0 \cdot 02$ \\
\hline $\begin{array}{l}\text { Melanosis on rectal } \\
\text { biopsy }(n)\end{array}$ & $1 / 5$ & $0 / 20$ & NS \\
\hline $\begin{array}{l}\text { Non-specific inflammatory } \\
\text { changes on rectal biopsy } \\
\text { (n) }\end{array}$ & $4 / 5$ & $12 / 20$ & NS \\
\hline Hypokalaemia (n) & $1 / 7$ & $4 / 40$ & NS \\
\hline $\begin{array}{r}\text { Radiological signs of } \\
\text { cathartic colon }(n)\end{array}$ & $1 / 7$ & $1 / 38$ & NS \\
\hline
\end{tabular}

NS denotes not significant.

*Stool consistency was graded into four categories: formed $=1$; soft $=2$; viscous $=3$; watery $=4$.

In patients with a positive urine test the possibility of interference from any non-laxative medication the patients were known to take at the time of the screening episodes was ruled out by a TLC of these drugs.

Organic disease was eventually excluded in 116 out of the 200 patients evaluated for the study. A final diagnosis could not be established in seven (five women) of the 40 patients tested for laxatives with negative results.

Table 1 compares various characteristics of patients stratified according to the result of the screening test. The two groups were comparable with regard to age, length of history, and reported characteristics concerning nocturnal diarrhoea, maximal number of bowel movements per day, and stool consistency. Patients with a positive test tended to have larger daily faecal weights (523 $v 297 \mathrm{~g} /$ day $)$, but this difference was not statistically significant. A history of psychiatric disorders was found significantly more often in patients with a positive test, and we confirmed the well known female preponderance of laxative abuse (Table 1).

A survey of potentially preventable costs in the seven patients with a positive screening test is given in Table 2. These patients spent 35 days in hospital worth DKK 88515, and were seen at 29 visits in the outpatient clinic (DKK 19633) after the time when a laxative screening test was found positive. Furthermore, diagnostic procedures worth a total of DKK 24371 were done in the same period of time 
(Table 2). The total cost of the laxative screening programme was DKK 20790 (99 urine analyses of DKK 186 and 18 stool water analyses of DKK 132).

\section{Discussion}

In this study the prevalence of surreptitious laxative consumption was registered in consecutive, unselected patients with diarrhoea of uncertain origin. We found that $15 \%$ of patients seeking medical help for diarrhoea in a university hospital gastroenterological clinic ingest laxatives, even though they deny doing so.

The prevalence rate may be even higher as seven of $54(13 \%)$ of the patients with proved diarrhoea were not screened for various reasons. Of special interest are four women who refused to participate in the study, when they learned that their excretions would be examined for laxatives.

In a Dutch study 15 of 81 patients $(19 \%)$, suspected of laxative abuse over a three year period, were definitely shown to use selfprescribed laxatives. Testing was performed for evidence of colonic stimulants in urine only.

Read et al" studied 27 patients over a six year period. The patients were referred with severe chronic diarrhoea, and all had previously received extensive diagnostic evaluation. Seven of these patients, all women, surreptitiously ingested laxatives, and this was the most common diagnosis that could be established. Within a five year period 17 cases of surreptitious laxative use were found at a large American referral centre with a 1000 bed hospital." This apparent low frequency was, at least in part, explained by the fact that patients suspected of laxative abuse were only tested for evidence of phenolphthalein ingestion.

In these studies ${ }^{511}$ only patients suspected of laxative abuse were tested, and no data were given concerning the total number of patients with unexplained diarrhoea, who were not tested for evidence of laxative consumption, thus impeding a direct comparison to our data.

The decision to examine a patient for a suspected abuse of laxatives is often provoked by the negative outcome of a comprehensive diagnostic programme, or by the finding of a clinical sign suggestive of laxative consumption, such as melanosis coli or a 'cathartic' colon. These morphologic changes cannot, however, be taken as evidence of a continued use of laxatives.

We found no single clinical feature in our patients that could have predicted the positive outcome of the test, but the combination of female sex and a history of psychiatric disorders was highly suggestive (Table 1).

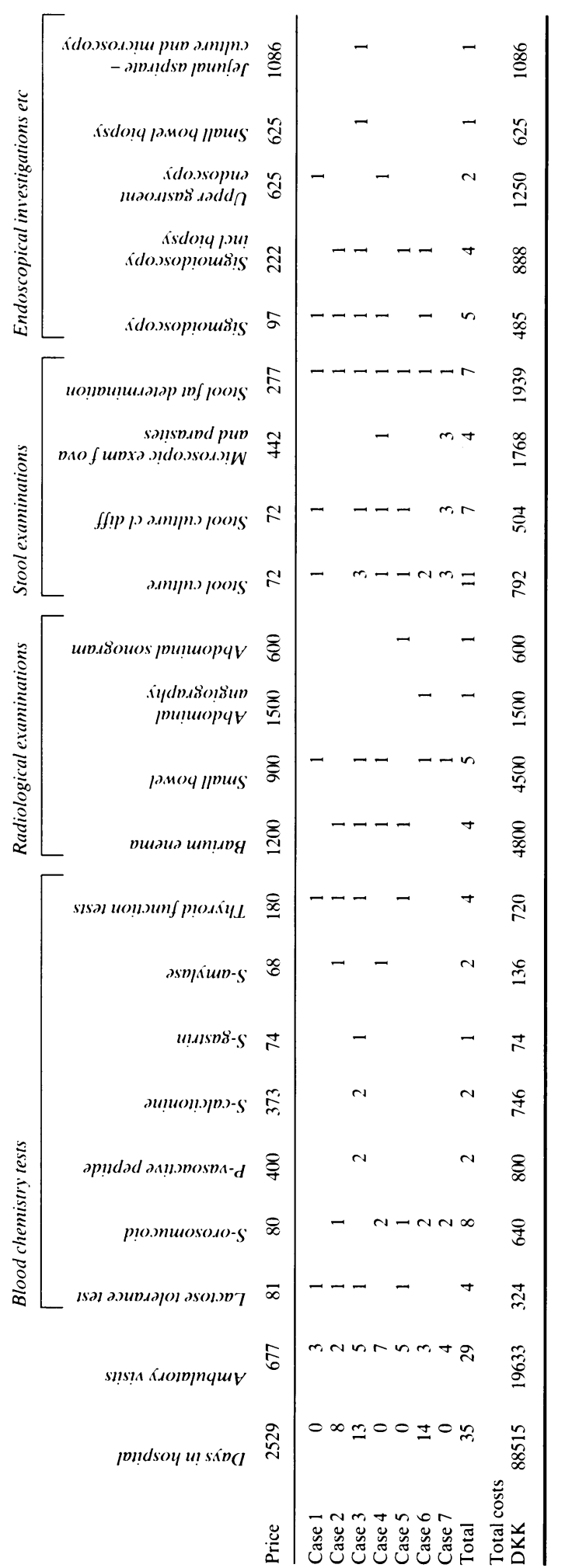


Detection of laxatives in urine or faeces is the most efficient way to establish a suspected diagnosis of laxative abuse beyond doubt. ${ }^{45}$ Repeated analyses are necessary as patients may use laxatives intermittently." Thus, five of seven urine specimens in one of the laxative abusers were normal, and a correct diagnosis may not have been reached by a single testing of this patient.

Because the majority of the patients in this study were only tested twice, we cannot exclude the possibility that we have underestimated the real prevalence of concealed laxative abuse. In most of these patients, however, the laxative screening procedure was stopped either because a diagnosis was found or because the diarrhoea ceased.

The diversity of laxative preparations makes it very cumbersome to establish a clinically relevant screening procedure, and we have previously suggested the addition of an indicator - for example, the free anthraquinone danthron, which is easily detected in urine, to all registered laxatives. ${ }^{+}$In this way the screening process might be simplified, although an analysis of faecal water would still be recommended in special cases in order to reveal patients who deliberately add tap water or urine to their stools to increase their bulk. ${ }^{x}$ Furthermore, non-registered laxatives often contain large amounts of highly effective purgatives and a screening for the various chemical principles (usually anthraquinones) is still the best way to establish the surreptitious intake of these cathartics that are sold without any pharmaceutical control.

Before a final decision of establishing this laxative screening test as an early, routine test in all patients with diarrhoea of uncertain origin, we wanted to estimate the possible costs and medical benefits of this diagnostic strategy. The potential cost savings of introducing the test were estimated by keeping the results secret to the clinicians. The costs of specific diagnostic procedures and the number of days in hospital and visits to the outpatient clinic that were redundant in patients with positive tests could then be calculated. In evaluating these figures, summarised in Table 2, it is important to recognise that some of the patients had been carefully investigated before being referred to our hospital. The cost of these tests are not potentially redundant, and consequently have not been registered.

Given a prevalence rate of $15 \%$ it is obvious that the screening programme is very cost effective, at least in a narrow monetary sense of the term. ${ }^{12}$ Thus, the price for screening the whole population of patients with diarrhoea of uncertain origin is more than balanced by what can be saved in specific diagnostic procedures in the laxative abusers.

In evaluating the possible medical benefits of the screening strategy, one must recognise that in four of the seven patients the participating clinicians did not suspect self-induced diarrhoea. Therefore, the correct diagnosis was missed or postponed in the normal diagnostic strategy, where a chemical detection of laxatives is restricted to cases in which clinical findings specifically suggest factitious diarrhoea. Consequently, some of the patients detected here did not show the classical features of the syndrome. '

Even though the treatment of patients with laxative abuse is generally unsuccessful, and follow up has shown relatively poor results, ${ }^{11}{ }^{13}$ most clinicians would agree that establishing the diagnosis early is an overall benefit to the patient, who may then be spared further unpleasant or risky investigations. In that sense the screening programme proved beneficial, as these patients could have been spared the relative risks of 10 roentgenographic contrast investigations (four barium enemas, five barium meals with follow through and one abdominal angiography) and 13 investigations involving intubation of the gastrointestinal tract (nine sigmoidoscopies, two upper endoscopies, one small bowel biopsy, and one jejunal aspirate) (Table 2).

We conclude that in at least $15 \%$ of unselected patients with diarrhoea of uncertain origin the bowel disorder can be explained by surreptitious laxative intake. We recommend the use of a comprehensive, early laxative screening programme of all patients with diarrhoea of uncertain origin as a cost beneficial and cost effective procedure.

The authors thank Grethe Eskesen for her help in collecting the urine and faecal water specimens.

\section{References}

1 Cummings JH. Laxative abuse. Gut 1974; 15: 758-66.

2 Cooke WT. Laxative abuse. Clin Gastroenterol 1977; 6: 659-73.

3 Ewe K, Karbach U. Factitious diarrhoea. Clin Gastroenterol 1986; 15: 723-40.

4 Bytzer P, Klitgaard NA. Chromatographic demonstration of anthraquinone derivatives in the urine. A method of proving surreptitious laxative abuse. Ugeskr Lager 1986; 148: 1390-2.

5 de Wolff FA, Edelbroek PM, de Haas EJM, Vermeij P. Experience with a screening method for laxative abuse. Human Toxicol 1983; 2: 385-9.

6 Plumeri PA. The room search. J Clin Gastroenterol 1984; 6: 181-5.

7 Cummings JH, Sladen GE, James OFW, Sarner M, Misiewicz JJ. Laxative-induced diarrhoea: a continuing clinical problem. Br Med J 1974; i: 537-41.

8 Morris AI, Turnberg LA. Surreptitious laxative abuse. Gastroenterology 1979; 77: 780-6. 
9 Read NW, Krejs GJ, Read MG, Santa Ana CA. Morawski SG, Fordtran JS. Chronic diarrhoea of unknown origin. Gastroenterology 1980; 78: 264-71.

10 de Wolff FA, de Haas EJM, Verweij M. A screening method for establishing laxative abuse. Clin Chem 1981; 27: 914-7.

11 Slugg PH, Carey WD. Clinical features and follow-up of surreptitious laxative users. Cleve Clin Q 1984; 51: 16771.

12 Doubilet $\mathrm{P}$, Weinstein MC, McNeil BJ. Use and misuse of the term 'cost effective' in medicine. $N$ Engl $J$ Med 1986; 314: 253-6.

13 Case Records of the Massachusetts General Hospital (Case 47-1985). N Engl J Med 1985; 313: 1341-6. 